\title{
Thromboelastographic changes during laparoscopic fundoplication
}

\author{
Indre Zostautiene ${ }^{1}$, Kristina Zvinienè ${ }^{1}$, Darius Trepenaitis², Rolandas Gerbutavičius ${ }^{3}$, Antanas Mickevičius ${ }^{4}$, \\ Rima Gerbutavičiené ${ }^{5}$, Mindaugas Kiudelis ${ }^{4}$ \\ ${ }^{1}$ Clinic of Radiology, Medicine Academy, Lithuanian University of Health Sciences, Kaunas, Lithuania \\ ${ }^{2}$ Clinic of Anesthesiology, Medicine Academy, Lithuanian University of Health Sciences, Kaunas, Lithuania \\ ${ }^{3}$ Clinic of Oncology and Haematology, Medicine Academy, Lithuanian University of Health Sciences, Kaunas, Lithuania \\ ${ }^{4}$ Clinic of Surgery, Medicine Academy, Lithuanian University of Health Sciences, Kaunas, Lithuania \\ ${ }^{5}$ Department of Drug Technology and Social Pharmacy, Lithuanian University of Health Sciences, Kaunas, Lithuania
}

Videosurgery Miniinv 2017; 12 (1): 19-27

DOI: https://doi.org/10.5114/wiitm.2017.66474

\begin{abstract}
Introduction: Thromboelastography (TEG) is a technique that measures coagulation processes and surveys the properties of a viscoelastic blood clot, from its formation to lysis.

Aim: To determine the possible hypercoagulability state and the effect of antithrombotic prophylaxis on thromboelastogram results and development of venous thrombosis during laparoscopic fundoplication.

Material and methods: The study was performed on 106 patients who were randomized into two groups. The first group received low-molecular-weight heparin (LMWH) $12 \mathrm{~h}$ before the operation, and 6 and $30 \mathrm{~h}$ after it. The second group received LMWH only $1 \mathrm{~h}$ before the laparoscopic fundoplication. The TEG profile was collected before LMWH injection, $1 \mathrm{~h}$ after the introduction of the laparoscope and $15 \mathrm{~min}$ after the surgery was completed.

Results: There was no significant difference in thromboelastography R-time between the groups before low-molecular-weight heparin injection. In group I preoperative R-values significantly decreased $1 \mathrm{~h}$ after the introduction of the laparoscope, after the end of surgery and on the third postoperative day. K-time values decreased significantly on the third postoperative day compared with the results before low-molecular-weight heparin injection, and after the operation. In group II, preoperative R-values significantly decreased $1 \mathrm{~h}$ after the introduction of the laparoscope, and after surgery. K-time values did not change significantly during or after the laparoscopic operation.

Conclusions: Our study results demonstrated that the hypercoagulation state (according to the TEG results) was observed during and after laparoscopic fundoplication in patients when LMWH was administered $12 \mathrm{~h}$ before the operation together with intraoperative intermittent pneumatic compression. The optimal anticoagulation was obtained when LMWH was administered $1 \mathrm{~h}$ before fundoplication.
\end{abstract}

Key words: laparoscopic fundoplication, thromboelastogram, hypercoagulability.

\section{Introduction}

Venous thromboembolism is an important social and health care problem, because $20-30 \%$ of patients develop deep vein thrombosis (DVT) after general surgical operations, while $5.5 \%$ of patients have this complication when laparoscopic fundoplication is performed without appropriate prophylaxis [1]. The most frequent reason for pulmonary embolism is thrombi forming in the channels of proximal leg veins and deep pelvis veins. Eighty-ninety percent of pulmonary embolism masses are caused by DVT or a thrombus formed in the pelvis [2]. The development of deep venous thrombosis is related to sta-

\section{Address for correspondence}

Indre Zostautiene, Clinic of Radiology, Medicine Academy, Lithuanian University of Health Sciences, Eiveniu g. 2 A, 50009 Kaunas,

Lithuania, phone: +370 600 70799, e-mail: indrajoss@gmail.com 
sis, hypercoagulability, and injury of the venous wall (Virchow's triad).

Laparoscopic surgery causes variable serum hypercoagulability; there are data suggesting that dependent positioning in combination with intraoperative pneumoperitoneum decreases venous flow from the lower extremities and possibly increases the risk of DVT development [3].

The increased intra-abdominal pressure associated with pneumoperitoneum and reverse Trendelenburg position during laparoscopic fundoplication generates venous stasis in the lower limb by compressing the retroperitoneal vena cava and iliac veins, which is already present due to general anesthesia [4].

The intermittent pneumatic compression of the calf with an external pressure cuff for the prevention of DVT is a well-established prevention measure [5-8]. Methods that have conventionally used to prevent postoperative deep vein thrombosis during laparoscopic surgery include not only mechanical techniques, but also drug therapy (low-molecularweight-heparin - LMWH). Some uncertainties are still present regarding $\mathrm{LMWH}$ administration time before elective surgery. Some guidelines suggest LMWH prophylactic administration $12 \mathrm{~h}$ before surgery, others $1-2 \mathrm{~h}$ before the operation and even 4-6 h after surgery. It is crucial to select the optimal time of LMWH administration in order to obtain the best therapeutic ratio for thrombosis prevention and avoiding excessive bleeding. For this reason, it is important to examine the effect of $\mathrm{LMWH}$ on some parameters of coagulation as surrogate markers of possible thrombosis development during and after surgery as it will help to guide physicians with the best time of anticoagulant administration. We chose the thromboelastogram in this respect because it is easy to obtain global coagulation assay with efficacy to detect effects of anticoagulants in various clinical scenarios. Thromboelastography (TEG) analysis is more sensitive to qualitative defects in fibrin or platelets than standard laboratory tests [4]. The technique of thromboelastograph hemostasis analysis provides a measurement of dynamic changes in the viscoelasticity property of the blood clot and gives permanent graphic documentation (Figure 1) [9]; TEG tracing provides all phases of the hemostasis process from the formation of the first fibrin strands to the fibrinolytic dissolution of the clot [4].

\section{Aim}

The aim of this study was to evaluate whether TEG parameters are able to detect the hypercoagulability state in patients undergoing laparoscopic fundoplication and to monitor the effect of the antithrombotic prophylaxis before, during and after laparoscopic fundoplication.

\section{Material and methods}

This was a prospective randomized clinical study, where 106 patients undergoing elective laparoscopic
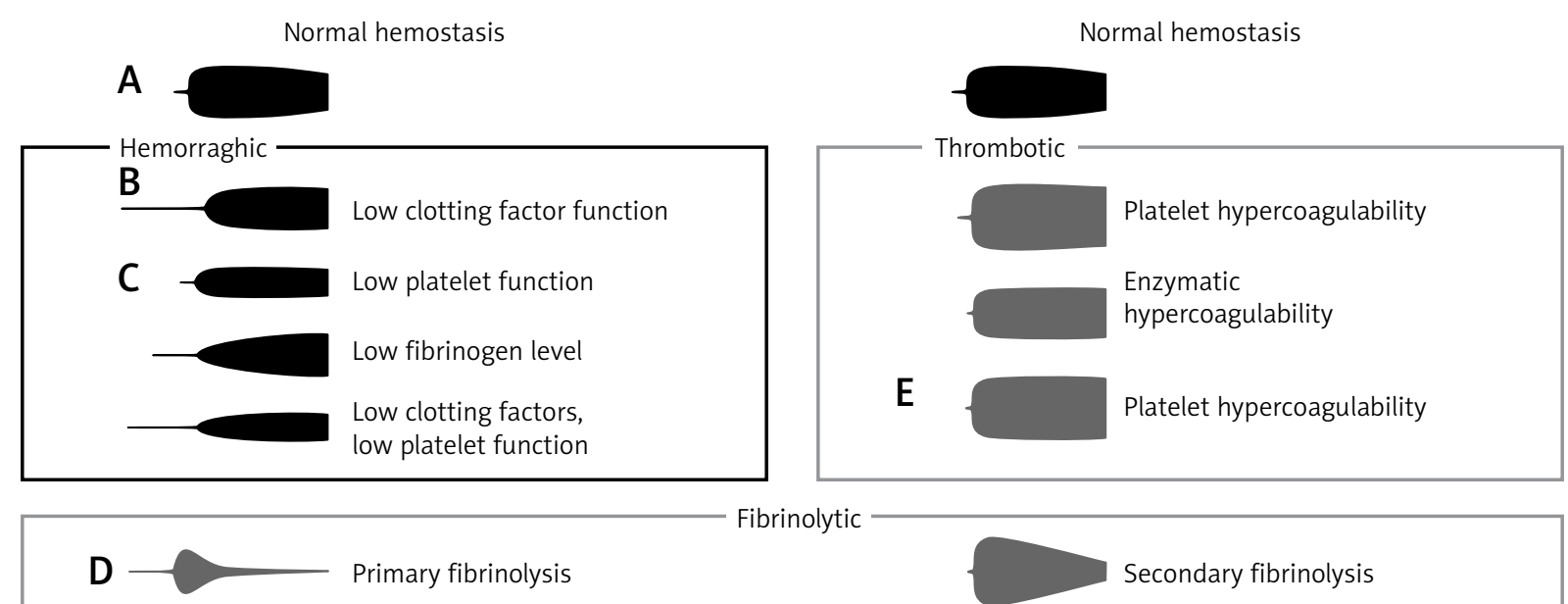

Secondary fibrinolysis

Figure 1. Characteristic thromboelastographic (TEG) tracings. A - Normal TEG tracing. B - Anticoagulation caused by coagulation factor deficiency or inhibition leading to a prolonged R time. C - Platelet dysfunction or pharmacological inhibition leading to a decreased maximum amplitude (MA). D - Hyperfibrinolysis. E - Hypercoagulability leading to shortened R and K times, with a concomitant elevated and angle 
fundoplication because of gastroesophageal reflux disease, caused by hiatal hernia, were studied. All the patients gave their written informed consent, and The Kaunas Regional Biomedical Research Ethics Committee approved the study (protocol no. BE-2-13). This randomized clinical study was also registered on the ISRCTN registry with trial ID ISRCTN62203940. All the patients were randomized into two groups (Figure 2). The first group of 55 patients received LMWH Bemiparinum (Zibor, Berlin Chemie, Luxembourg) 2500TV $0.2 \mathrm{ml}$ subcutaneously $12 \mathrm{~h}$ before the operation, and 6 and $30 \mathrm{~h}$ after it. The second group of 51 patients received LMWH Bemiparinum 2500 TV 0.2 ml. subcutaneously $1 \mathrm{~h}$ before the operation. Both groups received intermittent pneumatic compression (IPC) during all laparoscopic fundoplication. The IPC was performed using "Kendall SCD 700 Series" apparatus. We acquired 5-mm-thick axial CT (GE Light Speed Pro) venograms from the ankle to the iliac wing after injection of $150 \mathrm{ml}$ of $300 \mathrm{mg} / \mathrm{ml}$ contrast medium at a flow rate of $3 \mathrm{ml} / \mathrm{s}$ through an antecubital vein on the third postoperative day.

\section{Anesthesia}

All operations were performed under standardized endotracheal anesthesia. Premedication: all patients received $7.5 \mathrm{mg}$ of midazolam $1 \mathrm{~h}$ before transfer to the operating room. In the operating room standard monitoring was established including an electrocardiogram (five electrode system), peripheral oxygen saturation, and non-invasive blood pressure ("S/5 Compact" Datex-Ohmeda). Preoxygenation with $100 \%$ oxygen via a face mask for 3 min was followed by standardized induction of anesthesia with thiopentone $4-6 \mathrm{mg} / \mathrm{kg}$, fentanyl $2.5 \mu \mathrm{g} / \mathrm{kg}$, and pipecuronium $0.08 \mathrm{mg} / \mathrm{kg}$, and anesthesia was subsequently maintained with isoflurane $0.6-0.7$ of minimal alveolar concentration and fentanyl $1.0-1.25 \mu \mathrm{g} / \mathrm{kg}$ every $20-25 \mathrm{~min}$. Lungs were artificially ventilated with "S/5 Aespire" Datex-Ohmeda $\left(\mathrm{FiO}_{2} 0.40-0.45\right)$, respiration was monitored with "S/5 Compact" Datex-Ohmeda, and parameters of artificial lung ventilation were changed according to readings of capnography, keeping end tidal partial pressure of $\mathrm{CO}_{2}$ at $35-40 \mathrm{~mm} \mathrm{Hg}$. Neuro-

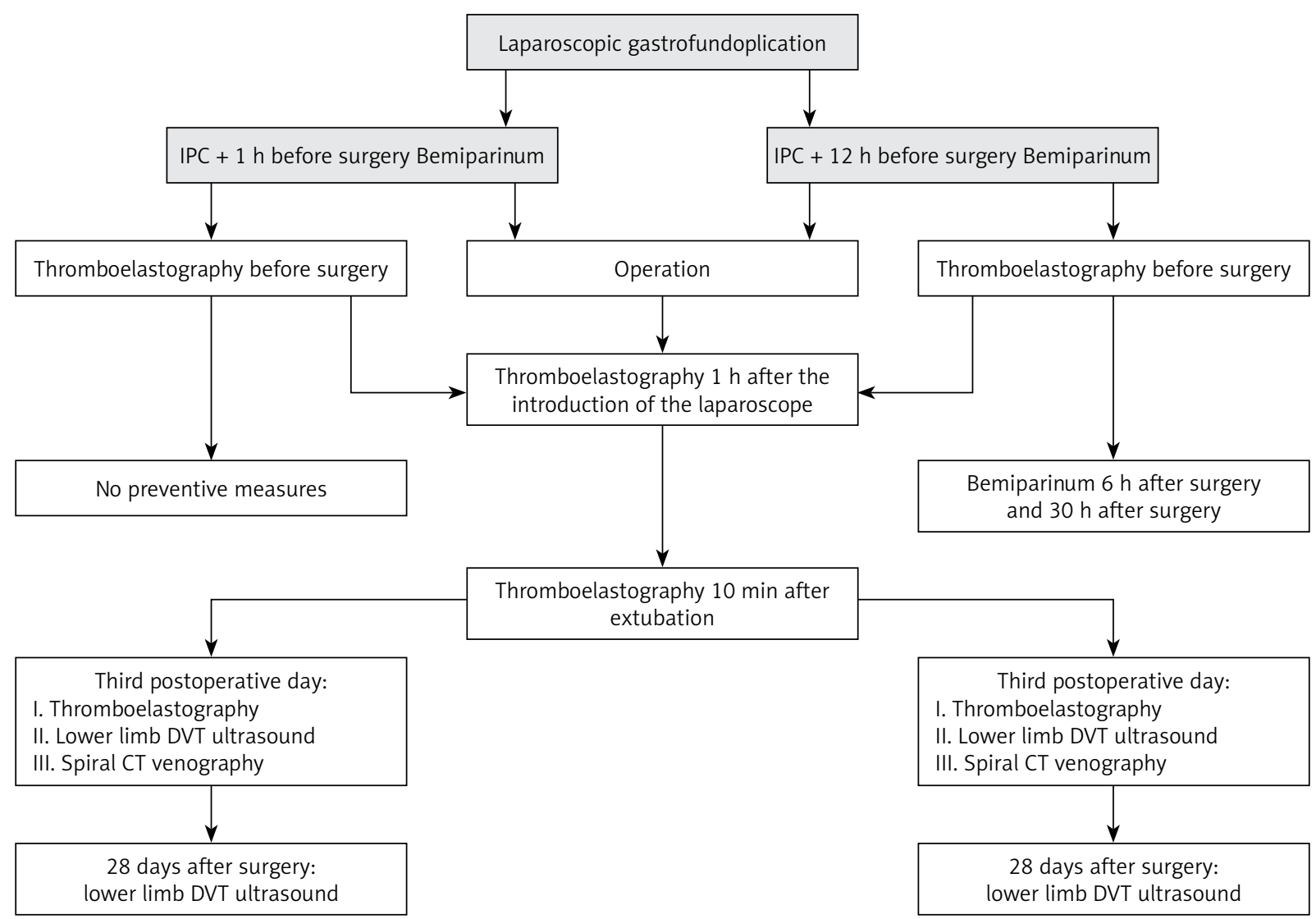

Figure 2. Study design 
muscular blockade was reversed by atropine $1.0 \mathrm{mg}$ and neostigmine $2.0 \mathrm{mg}$. All patients were extubated in the operating room. Infusion therapy: before insufflation all patients received intravenously $500 \mathrm{ml}$ of Ringer's solution; the total amount of fluid during the operation was $1500 \mathrm{ml}$ of Ringer's solution.

\section{Operation}

Two experienced surgeons performed all the operations. Pneumoperitoneum was induced by inserting a Veress needle above the umbilicus and connecting it to the $\mathrm{CO}_{2}$ insufflator, which achieved and maintained an intraabdominal pressure of $12-14 \mathrm{~mm} \mathrm{Hg}$. The patients were moved to the reverse Trendelenburg position $\left(45^{\circ}\right)$ before intraabdominal manipulation.

Five trocars were inserted into the peritoneal cavity at the typical sites - at the epigastrium, in the right subcostal area, two in the left subcostal area, and one on the middle abdominal line above the navel. The short gastric arteries were divided; the same procedure was performed on the gastric diaphragm ligament, releasing the gastric fundus. On the side of the small gastric curve, the liver-stomach ligament was cut, and the right diaphragm leg was prepared, followed by the left one. The mobilization of the esophagus was completed through bypassing it in the circular manner, freeing a 3-4 cm-long intraabdominal segment and removing the hernia

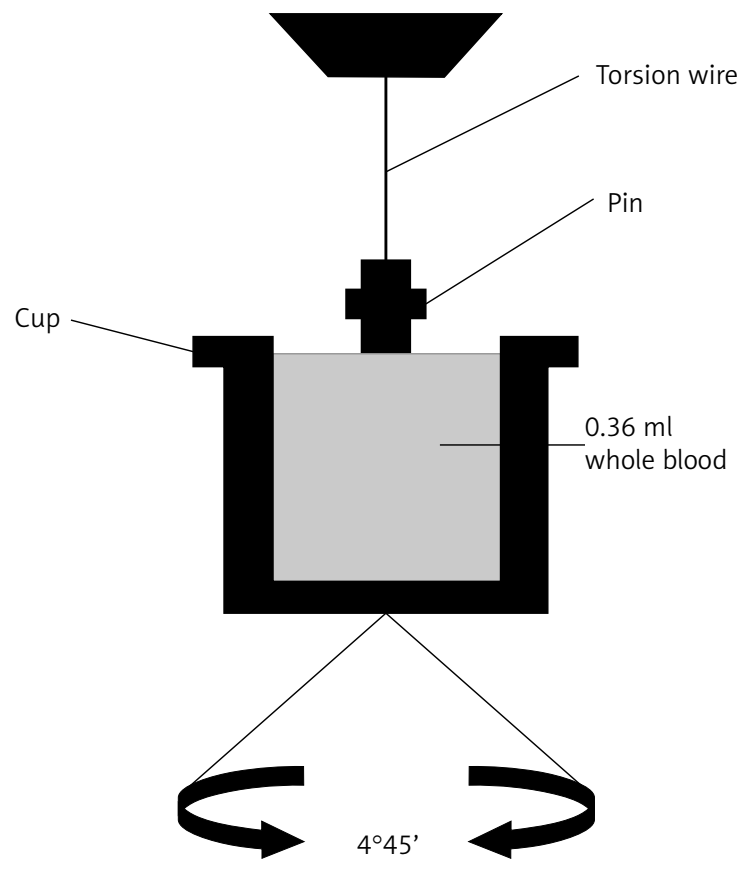

Figure 3. Technique to perform the TEG test sack. The diaphragm legs were then sutured using a 3-4-suture non-resorbing Ethibond (Ethicon, Johnson \& Johnson Company, USA) behind the esophagus. The next step of surgery was the formation of a $360^{\circ}$ (Nissen) antireflux wrap. It was formed by rolling a $2.5 \mathrm{~cm}$ long wrap of gastric fundus behind the abdominal portion of the esophagus, thus reinforcing the lower esophageal sphincter. The wrap was then fixed to the esophagus and the stomach was sutured to the front of the esophagus.

\section{Blood collection and processing}

Venous blood samples for TEG analysis were taken from the forearm vein four times:

1) before $L M W H$ injection an before the operation;

2) $1 \mathrm{~h}$ after the introduction of the laparoscope;

3) 15 min after extubation;

4) on the third postoperative day.

Blood was drawn by a clean venipuncture following a double-syringe technique and placed into vacutainer tubes containing 3.9\% (0.129 M) trisodium citrate (Becton Dickinson Vacutainer Systems, Plymouth, UK) in the ratio of one part of citrate to nine parts of whole blood. Whole blood was transferred within $5 \mathrm{~min}$ to prewarmed cuvettes of the thromboelastograph.

The TEG analysis was performed with TEG 5000 Thromboelastograph Hemostasis Analyzer system (Haemonetics Corporation, UK). The coagulation process was activated using Kaolin and $20 \mu \mathrm{l}$ of calcium chloride as described in the literature. The TEG system's approach to the monitoring of patient hemostasis is based on the premise that the end result of the hemostasis process is a single product, which is the clot [4]. The TEG system measures the clot's physical properties by the use of a cylindrical cup that holds the $0.36 \mathrm{ml}$ blood as it oscillates through an angle of $4^{\circ} 45^{\prime}$ and each rotation lasts for $10 \mathrm{~s}$ (Figure 3). As the clot begins to form in the presence of the activator, the TEG records the visceroelastic changes that occur in the sample.

A pin suspended in the blood within a stationary cup is monitored for motion as fibrin-platelet bonding occurs. This motion is related to the strength of the clot and is analyzed by a computer, which creates a graphical representation.

The TEG is a point-of-care whole blood coagulation monitor which provides information on specific aspects of coagulation including time to production of initial fibrin strands (R-time), time to develop a clot (R-time, K-time), rate of fibrin build-up and 
cross linking (a-angle), maximum clot strength (maximum amplitude - bMA) and measures of fibrinolysis (decreasing amplitude post-MA) [10] (Figure 4).

The thromboelastographic trace was analyzed for:

- R-time (reaction time, in minutes) - it is time from the beginning of the trace until an amplitude of $2 \mathrm{~mm}$ is reached. The period of latency time is taken from the placement of the blood sample in the cuvette to the initial fibrin formation.

- K-time (clotting time, in minutes) - it is the time from the end of $\mathrm{R}$ until a fixed clot strength is reached, i.e. amplitude of the trace is $20 \mathrm{~mm}$.

- \&-angle (clot formation rate) - it is affected primarily by the rate of thrombin generation.

- MA (maximum amplitude) - it is a measure of maximum strength of the clot and assesses the function of the platelets and to some extent fibrinogen also.

\section{Deep vein thrombosis diagnosis}

All the patients underwent color duplex scan examination preoperatively and spiral CT venography with color duplex scan on the third postoperative day in order to detect possible DVT. One experienced radiologist performed all these examinations. Criteria for DVT diagnosis were: intraluminal filling defect or localized nonopacification of the venous segment.

\section{Statistical analysis}

Statistical evaluation was conducted using descriptive analysis: normality test (Shapiro-Wilk W-test), the unpaired Mann-Whitney $U$ test to com-

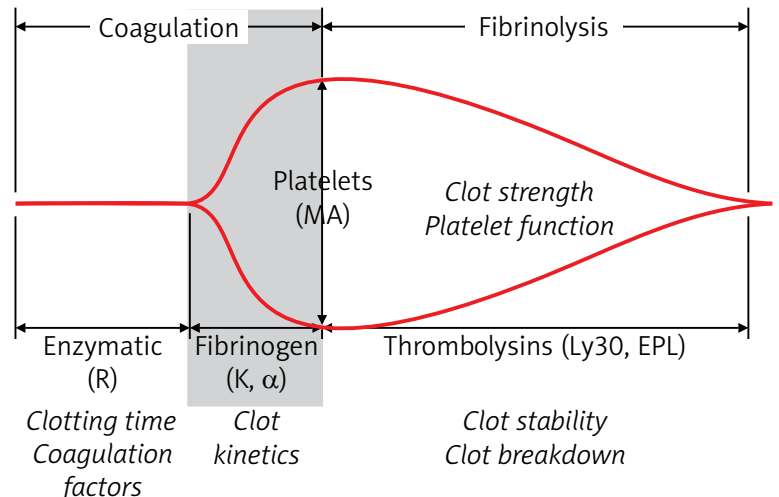

Figure 4. TEG tracing - normal values of TEG parameters (celite activated whole blood)

R-time - reaction time, in minutes (norm: 5.0-10.0 min) - this denotes the time taken from the beginning placement of the blood sample in the cuvette to the initial fibrin formation. K-time - clotting time (norm; 1.0-3.0 $\mathrm{min}$ ) representing the time of fixed clot formation. \&-angle - degrees; closely related to K (norm: 53.0$\left.72.0^{\circ}\right)$ this represents the rate of clot growth. MA parameter maximum amplitude, millimeters (norm: 50.0-70.0 mm) - a measurement of maximum strength of the developed clot.

pare data from two study groups, and the Wilcoxon test to investigate differences in TEG values in each group of patients. Data are expressed as mean \pm SD. $P$-value $<0.05$ was considered significant.

\section{Results}

The patients in both groups were similar in terms of age, weight, height, gender, duration of surgery, and American Society of Anesthesiologists (ASA) class (Table I). There was no massive or minor bleeding during all laparoscopic operations. No drains

Table I. Patients' demographic characteristics

\begin{tabular}{|lccc|}
\hline Value & Group I $(N=55)$ & Group II $(N=51)$ \\
\hline Age [years] & $55.27 \pm 14.25$ & $55.24 \pm 14.65$ & $16 / 35$ \\
\hline Male/female $(n / n)$ & $18 / 37$ & $26.72 \pm 4.82$ & 0.886 \\
\hline BMI $\left[\mathrm{kg} / \mathrm{m}^{2}\right]$ & $27.03 \pm 5.04$ & $129.71 \pm 36.84$ \\
\hline Duration of surgery [min] & $136.73 \pm 45.16$ & 6 \\
\hline ASA class: & 7 & 30 \\
\hline I & 29 & 0.638 \\
\hline II & 18 & 0.921 \\
\hline III & 1 & 0.921 \\
\hline IV & $3.98 \pm 0.913$ & 0.921 \\
\hline Postoperative stay [days] & & 0.661 \\
\hline
\end{tabular}



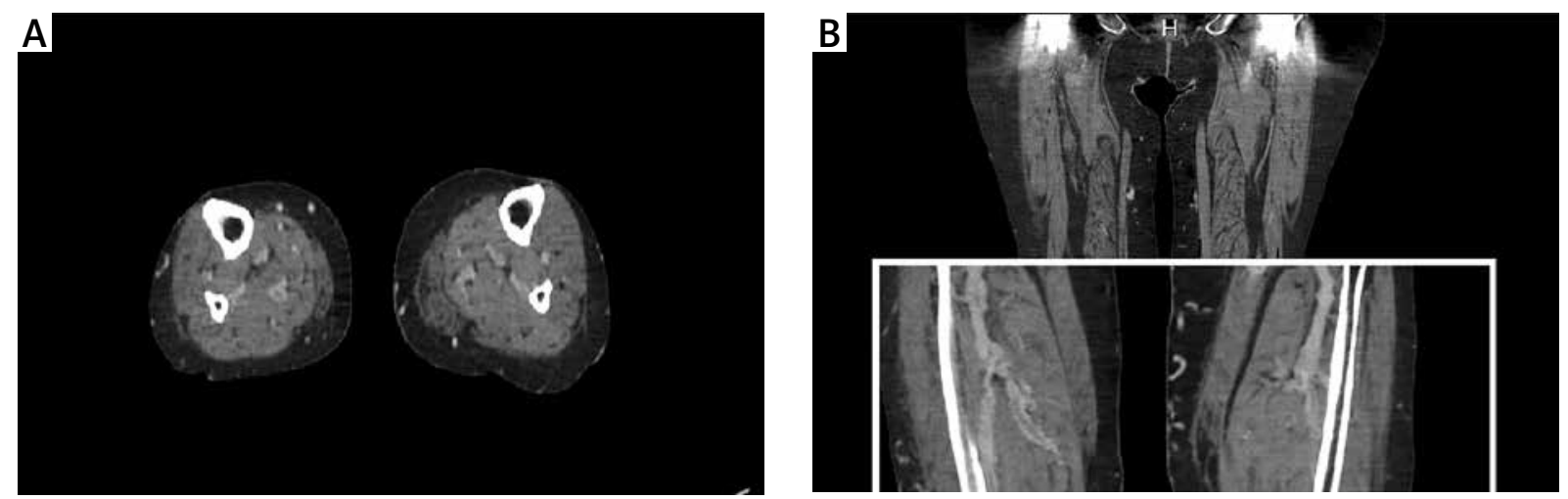

Photo 1. Computed tomography venography revealed v. poplitea dex. thrombosis: A - transverse CT scan, B - coronal CT scan

were left after the operation. Computed tomography venography revealed popliteal vein thrombosis in 2 (3.6\%) group I patients on the third postoperative day (Photo 1). All the patients left the hospital after an uneventful 3-5-day stay.

TEG values - R-time, $K$-time, $\alpha$-angle and maximum amplitude - were not significantly different between the groups before LMWH injection, during and after laparoscopic fundoplication (Tables II-V).

In group I, preoperative TEG R-values significantly decreased already $1 \mathrm{~h}$ after the introduction of the laparoscope, and remained at the same level until the third postoperative day (Table II). TEG K-time values decreased significantly only on the third postoperative day compared with the results before LMWH injection, during the surgery or after the operation (Table III).

In group II, TEG R-time and K-time values did not change significantly during or after the laparoscopic operation (Tables II and III).

There were no significant changes in $\alpha$-angle and maximum amplitude values during or after laparoscopic fundoplication in both groups (Tables IV and $\mathrm{V}$ ).

Table II. R-time value changes in both groups

\begin{tabular}{|lcccc|}
\hline Study groups & Before LMWH injection & $\begin{array}{c}1 \mathrm{~h} \text { after introduction } \\
\text { of the laparoscope }\end{array}$ & After extubation & Third postoperative day \\
\hline I $(N=55)$ & $7.41 \pm 1.51^{\bullet \bullet \bullet}$ & $6.40 \pm 2.65^{\bullet}$ & $6.28 \pm 1.90^{\star \bullet}$ & $6.09 \pm 1.44^{\bullet}$ \\
\hline II $(N=51)$ & $6.36 \pm 1.84^{* \infty}$ & $5.10 \pm 1.64^{*}$ & $5.35 \pm 2.15^{\infty}$ & $5.87 \pm 2.12$ \\
\hline
\end{tabular}

$\cdot p=0.002,{ }^{*} p<0.001, \bullet p<0.001,{ }^{*} p=0.010,{ }^{\infty} p=0.001$. R-time $(5.0-10.0 \mathrm{~min})$.

Table III. K-time value changes in both groups

\begin{tabular}{|lcccc|}
\hline Study groups & Before LMWH injection & $\begin{array}{c}1 \mathrm{~h} \text { after introduction } \\
\text { of the laparoscope }\end{array}$ & After extubation & Third postoperative day \\
\hline I $(N=55)$ & $2.68 \pm 1.50^{*}$ & $2.59 \pm 1.97$ & $2.82 \pm 1.61$ & $2.22 \pm 1.04 \cdot \boldsymbol{\bullet}$ \\
\hline II $(N=51)$ & $2.13 \pm 1.29$ & $2.01 \pm 1.57$ & $2.12 \pm 2.25$ & $2.14 \pm 1.71$ \\
\hline
\end{tabular}

Table IV. Alpha-angle value changes in both groups

\begin{tabular}{|lcccc|}
\hline Study groups & Before LMWH injection & $\begin{array}{c}1 \mathrm{~h} \text { after introduction } \\
\text { of the laparoscope }\end{array}$ & After extubation & Third postoperative day \\
\hline I $(N=55)$ & $55.12 \pm 11.35$ & $55.88 \pm 14.02$ & $54.93 \pm 13.91$ & $57.25 \pm 12.14$ \\
\hline II $(N=51)$ & $59.34 \pm 13.81^{\bullet \bullet}$ & $65.24 \pm 12.68$ & $64.71 \pm 15.01 \bullet$ & $62.35 \pm 15.12$ \\
\hline
\end{tabular}

$p=0.010, \bullet p=0.012$. Alpha-angle (53.0-72.0 $)$. 
Table V. Maximum-amplitude value changes in both groups

\begin{tabular}{|lcccc|}
\hline Study groups & Before LMWH injection & $\begin{array}{c}1 \mathrm{~h} \text { after introduction } \\
\text { of the laparoscope }\end{array}$ & After extubation & Third postoperative day \\
\hline I $(N=55)$ & $64.86 \pm 8.97$ & $66.13 \pm 9.33$ & $63.17 \pm 8.20^{\bullet}$ & $65.98 \pm 7.29^{\bullet}$ \\
\hline II $(N=51)$ & $63.13 \pm 11.85$ & $61.11 \pm 22.29$ & $64.41 \pm 7.21$ & $66.20 \pm 7.03^{\bullet}$ \\
\hline
\end{tabular}

$\cdot p=0.015, \quad p=0.028$. Max. amplitude $(50.0-70.0 \mathrm{~mm})$.

\section{Discussion}

Thromboelastography is a viscoelastic hemostatic assay that measures the global visco-elastic properties of whole blood clot formation under low shear stress. Thromboelastography also is a method to monitor the hemostasis that is easy to use in the operation theatre, and the anesthesiologist is provided with rapid and accurate results. The formation of the clot is a net result of interaction between the cellular components of blood and coagulation proteins. The overall coagulation profile can be quantitatively and qualitatively interpreted in terms of the hypocoagulable, normal or hypercoagulable state of the sample and degree of lysis [11]. The TEG is a technique that gives a global overview of the coagulation cascade including fibrinolysis. Many workers have tried to correlate TEG parameters with the conventional coagulation profile, but it is not possible as the two techniques are different [11]. Commonly used blood tests are often aspecific, whereas the dosage of all plasma factors involved in coagulation and platelet activity is expensive and mostly not useful to individuate the clinical risk of hypercoagulability [12]. But to some extent, R correlates with activated partial thromboplastin time (APTT), and angle correlates with fibrinogen (and platelet function), MA correlates with platelet function (and fibrinogen) [13-18], and whole blood clot lysis index correlates with euglobulin lysis time and fibrin degradation products (FDP) [11].

It is already proven that laparoscopic surgery leads to postoperative activation of the coagulation system, which is a prerequisite for thromboembolic complications. Many factors can cause postoperative changes of hemostasis. These include the type of operation performed and the type of anesthesia, duration of the operation, and transfusion [19]. In laparoscopic surgery, the pneumoperitoneum reduces venous flow from the lower extremities by approximately $30 \%$ to $40 \%$ [19]. Therefore, the risk of
DVT might be increased after laparoscopic surgery compared with open surgery.

Topal et al. [19] showed that high intra-abdominal pressure led to a significant variation in hemostasis and coagulation compared with low intra-abdominal pressure. They used 10, 13 and $16 \mathrm{~mm} \mathrm{Hg}$ intra-abdominal pressure with the standard anesthetic technique in this study.

Conventional tests only monitor a single point of LMWH's effect on the coagulation cascade, whilst TEG can be used to assess the full anticoagulant effects of LMWH [20]. The LMWHs are unique in their ability to preferentially inhibit factor Xa more than factor Ila. Low-molecular-weight heparin has multiple potential effects on the coagulation pathway including antifactors IIa, IXa and Xa activity, inhibition of thrombin-induced activation of factors $\mathrm{V}$ and VIII, platelet function inhibition, and enhanced fibrinolysis and endothelial secretion of tissue factor pathway inhibitor [20-22]. Klein et al. [22] reported that thromboelastographic $R$ value correlates significantly with the peak and trough levels of anti-Xa activity after the administration of $\mathrm{LMWH}$.

Anti-factor Xa activity is not advised as a routine screening test as it does not reliably predict the risk of peri-operative bleeding [23, 24].

Our study compared the occurrence of DVT and thromboelastometric parameter changes during and after laparoscopic fundoplication with different pharmacologic prevention: low-molecular-weight heparin administered $1 \mathrm{~h}$ or $12 \mathrm{~h}$ before the surgery. Bemiparin is a LMWH indicated for the acute treatment of deep vein thrombosis with or without pulmonary embolism, for the prophylaxis of venous thromboembolism in surgical and nonsurgical patients and for the prevention of clotting in the extracorporeal circuit during hemodialysis [25]. Bemiparin is the second generation LMWH with the lowest molecular weight, the longest half-life [16] and the highest anti-factor $\mathrm{Xa}$ /anti-factor Ila activity ratio.

Our study results demonstrated that the low-molecular-weight heparin protected from possible oper- 
ation-induced hypercoagulation when it was administered $1 \mathrm{~h}$ before laparoscopic fundoplication - we found no difference in R- and K-time, $M A$, and $\alpha$-angle compared with baseline values in thromboelastographic examination. Despite the IPC action, when LMWH was administered $12 \mathrm{~h}$ before surgery, significant intraoperative and postoperative shortening of $\mathrm{R}$ time and $\mathrm{K}$ time on the third postoperative day and no difference in $\mathrm{MA}$ and $\mathrm{a}$-angle were detected. These findings suggests that the hypercoagulability state is present, which starts already during laparoscopic fundoplication and continues some days after it. These results of ours nicely correspond with LMWH pharmacokinetic data: bemiparin is rapidly absorbed after subcutaneous administration, attaining maximal plasma anti-Xa activity within 2-6 h. Postoperative administration of bemiparin ( 6 and $30 \mathrm{~h}$ after fundoplication) does not protect from intervention-induced hypercoagulation as R- and $\mathrm{K}$-time in this patient group remained shortened even on the third postoperative day. These results of ours demonstrate that prevention of operation-induced hypercoagulability is of crucial importance and it can be achieved with timely LMWH administration (one hour before laparoscopic surgery).

Caprini et al. [26] investigated TEG preoperatively and on the day following the operation in 100 laparoscopic cholecystectomy cases. They found significant postoperative shortening of $\mathrm{R}$ and $\mathrm{K}$ but no difference in MA. They suggested that postoperative coagulation asthenia should be attributed not to platelets but to coagulation activation. This was considered to reflex excessive enhancement of the activation of coagulation factors.

Forfori et al. [12] analyzed the correlation between the variation of normal TEG parameters and the dosage amount of anticoagulant therapy adjusted to body weight. They found no correlation between the variations of all screened TEG parameters from basal to postoperative and pro/kilogram dosage of $\mathrm{LMWH}$ administered. The authors suggest that LMWH prophylaxis based on weight produces a feasible but not a more efficient anticoagulation profile than standard LMWH therapy.

The exact incidence of thromboembolic complications after laparoscopic fundoplication is unknown, and we could not find studies on the dynamics of intraoperative and postoperative coagulation during laparoscopic fundoplications. Our study identified two cases of calf deep vein thrombosis (3.6\%) only in the group in which LMWH was administered $12 \mathrm{~h}$ before laparoscopic surgery. Keeping in mind that patients after laparoscopic fundoplication are mobile within a few hours after surgery, we can speculate that calf deep vein thrombosis in these two patients occurred during the operation. According our study results, we can also assume with caution that prophylaxis with intraoperative IPC and LMWH $12 \mathrm{~h}$ before surgery is not efficient enough to protect against the hypercoagulation state, present during laparoscopic fundoplication.

On the other hand, the main weakness of our study is that the number of patients is too small in both groups, so it is impossible to find a significant difference in the rate of DVT between the groups.

Venous stasis in laparoscopic operations can be minimized by reducing intraabdominal pressure during the operation. Combination of LMWH $1 \mathrm{~h}$ before laparoscopic surgery and intraoperative IPC generates a hypocoagulation effect more effective to prevent deep-vein thrombosis during or after the laparoscopic operation.

\section{Conclusions}

Monitoring TEG parameters can be a feasible practice to observe the coagulation status in patients undergoing laparoscopic surgery. Our first group patients presented a tendency to the hypercoagulability state, determined mostly by the shortening of TEG R-time. Comparison of TEG values between the study groups indicates that prophylaxis with $\mathrm{LMWH}$ $1 \mathrm{~h}$ before surgery together with IPC protects patients from surgery-induced hypercoagulation, and this lasts at least for three days. Our recommendation is that LMWH, as a DVT prophylactic measure, has to be administered $1 \mathrm{~h}$ before the laparoscopic operation to ensure the drug's optimal effect.

\section{Conflict of interest}

The authors declare no conflict of interest.

\section{References}

1. Kiudelis M, Gerbutavičius R, Gerbutavičienė R, et al. A combination effect of low-molecular-weight heparin and intermittent pneumatic compression device for thrombosis prevention during laparoscopic fundoplication. Medicina (Kaunas) 2010; 46: 18-23.

2. Houshmand S, Salavati A, Hess S, et al. The role of molecular imaging in diagnosis of deep vein thrombosis. Am J Nucl Med Mol Imaging 2014; 4: 406-25. 
3. Nguyen NT, Owings JT, Gosselin R, et al. Systemic coagulation and fibrinolysis after laparoscopic and open gastrib bypass. Arch Surg 2001; 136: 909-16.

4. Lee BY, Butler G, Al-Waili N, et al. Role of thrombelastograph haemostasis analyser in detection hypercoagulability following surgery with and without use of intermittent pneumatic compression. J Med Eng Technol 2010; 34: 166-71.

5. Sato H, Izuta S, Misumi T, et al. Incidence and clinical characteristics of perioperative pulmonary thromboembolism under the use of intermittent pneumatic compression as preventive measure. Masui 2003; 52: 1300-4.

6. Kiudelis M, Endzinas Z, Mickevicius A, et al. Venous stasis and deep vein thrombosis prophylaxis during laparoscipoic fundoplication. Zentralblatt fur Chirurgie 2002; 127: 944-9.

7. Labropoulos N, Cunningham J, Kang SS, et al. Optimising the performance of intermittent pneumatic compression devices. Eur J Vasc Endovasc Surg 2000; 19: 593-7.

8. Malone MD, Cisek PL, Comerota Jr, et al. High-pressure, rapid-inflation pneumatic compression improves venous hemodynamics in healthy volunteers and patients who are post-thrombotic. J Vasc Surg 1999; 29: 593-9.

9. Mauffrey C, Cuellar III DO, Pieracci F, et al. Strategies for the management of haemorrhage following pelvic fractures and associated trauma-induced coagulopathy. Bone Joint 2014; 96-B: 1143-54.

10. Ronald A, Dunning J. Can the use of thromboelastography predict and decrease bleeding and blood and blood product requirements in adult patients undergoing cardiac surgery? Interact Cardiovasc Thorac Surg 2005; 4: 456-63.

11. Narani KK. Thromboelastography in the perioperative period. Indian J Anaesth 2005; 49: 89-95.

12. Forfori F, Ferro B, Mancini B, et al. Role of thromboelastography in monitoring perioperative coagulation status and effect of thromboprophylaxis in bariatric surgery. Obes Surg 2012; 22: $113-8$.

13. Overview of the thrombelastograph coagulation analyzer. Haemoscope Corporation, Skokie, IL, USA.

14. Chandler WL. The thrombelastography and the thrombelastograph technique. Semin Thromb Hemost 1995; 21 Suppl. 4: 1-6.

15. Traverso Cl, Caprini JA, Arcelus J. The normal thrombelastogram and its interpretation. Semin Thromb Hemost 1995; 21: 7-13.

16. Oshita K, Az-ma T, Osawa Y, et al. Quantitative measurement of thromboelastography as a function of platelet count. Anesth Analg 1999; 89: 296-9.

17. Katori N, Szlam F, Levy JH, et al. A novel method to assess platelet inhibition by eptifibatide with thrombelastograph. Anesth Analg 2004; 99: 1794-9.

18. Tuman KJ, McCarthy RJ, Patel RV, et al. Comparison of thromboelastography and platelet aggregometry. Anesthesiology 1991; 75: A433

19. Topal A, Celik JB, Tekin A, et al. The effects of 3 different intra-abdominal pressures on the thromboelastographic profile during laparoscopic cholecystectomy. Surg Laparosc Endosc Percutan Tech 2011; 21: 434-8.

20. Casey ME, Hodges O, Dunn R, et al. Thromboelastography to monitor the intra-operative effects of low-molecular weight heparin following brodging antocoalation in a child with normal renal function. Anaesthesia 2013; 68: 91-6.

21. Backe SK, Lyons GR. High-dose tinzaparin in pregnancy and the need for urgent delivery. Br J Anaesth 2002; 89: 331-4.

22. Klein SM, Slaughter TF, Vail PT, et al. Thromboelastography as a perioperative measure of anticoagulation resulting from low molecular weight heparin: a comparison with anti-Xa concentrations. Anesth Analg 2000; 91: 1091-5.

23. Bounameaux H, de Moerloose P. Is laboratory monitoring of low-molecular-weight-heparin therapy necessary? J Thromb Haemost 2004; 2: 551-4.

24. Simons R, Mallet SV. Use of thromboelastography to demonstrate persistent anticoagulation after stopping enoxaparin. Anaesthesia 2007; 62: 1175-8.

25. Ciccone MM, Cortese Fr, Corbo F, et al. Bemiparin, an effective and safe low molecular weight hemiparin: a review. Vasc Pharmacol 2014; 62: 32-7.

26. Caprini JA, Arcelus JI, Laubach M, et al. Postoperative hypercoagulability and deep-vein thrombosis after laparoscopic cholecystectomy. Surg Endosc 1995; 9: 304-9.

Received: 29.12.2016, accepted: 11.01.2017. 